\title{
MicroRNA-30b is a multifunctional regulator of aortic valve interstitial cells
}

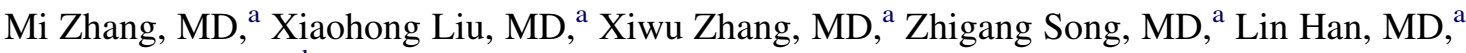 \\ Yuanyuan $\mathrm{He}, \mathrm{MD},{ }^{\mathrm{b}}$ and Zhiyun $\mathrm{Xu}, \mathrm{MD}^{\mathrm{a}}$
}

\begin{abstract}
Objective: Calcific aortic valve disease is an active process involving a wide range of pathologic changes. Valve interstitial cells are the most prevalent cells in the heart valve and maintain normal valve structure and function. MicroRNAs (miRNAs) are essential posttranscriptional modulators of gene expression, and miRNA-30b is a known repressor of bone morphogenetic protein 2-mediated osteogenesis. We hypothesized that miRNA$30 \mathrm{~b}$ is a multifunctional regulator of aortic valve interstitial cells during calcification.
\end{abstract}

Methods: To determine the role of miRNA-30b in calcific aortic valve disease, we evaluated miRNA expression in human calcific aortic valve leaflets obtained intraoperatively. Furthermore, human valve interstitial cells were evaluated with regard to miRNA-30b expression and osteogenesis by quantitative real-time polymerase chain reaction, Western blotting, flow cytometry, and alkaline phosphatase assays.

Results: In this study, we demonstrated that miRNA-30b attenuates bone morphogenetic protein 2-induced osteoblast differentiation by targeting Runx2, Smad1, and caspase-3. Transfection of a mimic of miRNA-30b led to decreases in alkaline phosphatase activity and expressions of Runx2, Smad1, and caspase-3. Furthermore, dual luciferase reporter assays confirmed that Runx2, Smad1, and caspase-3 are direct targets of miRNA-30b.

Conclusions: We demonstrated a remarkable role of miRNA-30b in calcific aortic valve disease as a regulator of human aortic valvular calcification and apoptosis through direct targeting of Runx2, Smad1, and caspase-3. Targeting of miRNA-30b could serve as a novel therapeutic strategy to limit progressive calcification in aortic stenosis. (J Thorac Cardiovasc Surg 2014;147:1073-80)

\section{Supplemental material is available online.}

Calcific aortic valve disease (CAVD) is a major health problem threatening aging societies. Once hypothesized to be the result of degenerative calcification, CAVD pathogenesis is now considered to be a complex and active process characterized by multiple pathologic changes. ${ }^{1-4}$ Valve interstitial cells (VICs) are the most prevalent cells in the heart valve and maintain normal valve structure and function. In diseased valves, however, VICs acquire an osteoblastlike phenotype during valve repair and

\footnotetext{
From the Department of Cardiothoracic Surgery, ${ }^{a}$ Changhai Hospital, Second Military Medical University, Shanghai, China; and Xinhua Hospital, ${ }^{\text {b }}$ Shanghai Jiaotong University School of Medicine, Shanghai, China.

This study was supported by the Key Basic Research Project of Shanghai titled, "The Role of miRNAs in Calcified Aortic Valve Disease," grant 11JC1415900.

Disclosures: Authors have nothing to disclose with regard to commercial support.

Received for publication Jan 21, 2013; revisions received May 4, 2013; accepted for publication May 9, 2013; available ahead of print Aug 21, 2013.

Address for reprints: Zhiyun Xu, MD, Department of Cardiothoracic Surgery, Changhai Hospital, Second Military Medical University, 174 Changhai Rd, Shanghai 200433, China (E-mail: zhiyunx@ @otmail.com).

0022-5223/\$36.00

Copyright (C) 2014 Published by Elsevier Inc. on behalf of The American Association for Thoracic Surgery

http://dx.doi.org/10.1016/j.jtcvs.2013.05.011
}

remodeling. Prevention of VIC transformation by blocking either cell differentiation or apoptosis may thus be beneficial in the prevention of CAVD. Until recently, however, the regulation of VIC function has been poorly understood.

MicroRNAs (miRNAs) bind to the $3^{\prime}$-untranslated region (UTR) in specific target messenger RNAs (mRNAs) and regulate their expression by inhibiting translation or promoting target degradation. ${ }^{5}$ Recent studies have indicated that miRNAs play an important role in osteogenic differentiation. $^{6,7}$ Previous experiments with MC3T3-E1 cells showed that miRNA-30 family members function as negative regulators of osteoblastic differentiation by targeting the master osteogenic transcription factors Smad1 and Runx $2 .^{8}$ The effect of miRNA is cell type-dependent, ${ }^{9}$ however, and the specific miRNAs and target genes involved in the regulation of human VIC calcification have not been well characterized.

To illustrate the role of miRNA-30s in CAVD, we demonstrated that miRNA-30b was the most dramatically downregulated miRNA-30 family member both in pathologic CAVD samples and in bone morphogenetic protein (BMP) 2-stimulated aortic VICs. Our gain and loss of function experiments confirmed that by binding to multiple sites in the 3'-UTRs of Runx2, Smad1, and caspase-3 mRNAs, miRNA-30b acts as an endogenous attenuator of these genes in VICs to antagonize heart valve calcification. 


$$
\begin{aligned}
& \text { Abbreviations and Acronyms } \\
& \text { ALP = alkaline phosphatase } \\
& \text { BMP = bone morphogenetic protein } \\
& \text { CAVD }=\text { calcific aortic valve disease } \\
& \text { FBS } \quad \text { fetal bovine serum } \\
& \text { I-miRNA-30b }=\text { microRNA-30b inhibitor } \\
& \text { miRNA }=\text { microRNA } \\
& \text { miRNA-NC }=\text { microRNA negative control } \\
& \text { M-miRNA-30b }=\text { microRNA-30b mimic } \\
& \text { mRNA }=\text { messenger RNA } \\
& \text { qRT-PCR = quantitative real-time } \\
& \text { polymerase chain reaction } \\
& \text { UTR = untranslated region } \\
& \text { VIC }=\text { valve interstitial cell }
\end{aligned}
$$

\section{METHOD AND MATERIALS \\ Reagents}

Dulbecco modified Eagle medium, minimum essential medium $\alpha$, and fetal bovine serum (FBS) were purchased from Gibco (Gibco; Invitrogen Corporation, Carlsbad, Calif). Bioactive recombinant human BMP-2, dexamethasone, ascorbic acid, and $\beta$-glycerophosphate were purchased from Sigma-Aldrich (Sigma, St Louis, Mo). Anti-Runx2, anti-Smad1, anti-phosphorylated Smad1/5/8 and antiosteocalcin antibodies were purchased from Santa Cruz Biotechnology (Santa Cruz Biotechnology, Inc, Santa Cruz, Calif). Anti-caspase-3 antibody was purchased from Cell Signaling Technology, Inc. (Cell Signaling Technology, Inc, Danvers, Mass). The miRNA-30b mimic (M-miRNA-30b), the miRNA-30b inhibitor (I-miRNA-30b), and an miRNA negative control (miRNA-NC) were synthesized by GenePharma (GenePharma Co, Ltd, Shanghai, China).

\section{Calcific Aortic Valve Collection}

Calcific aortic valve samples for evaluation of miRNA expression were collected from the 10 patients described in Table 1 who underwent aortic valve replacement. Exclusion criteria included congenital valve disease; nonstenotic, infective endocarditis; and rheumatic aortic valvulopathy. The study was approved by the Changhai Hospital ethics committee, and consent from the donors or their immediate family was obtained. After resection, the leaflets were sectioned into 2 parts. One part contained calcification nodules, and the other part consisted of surrounding tissues that served as controls. Tissues from the same patient were immediately frozen in pairs in liquid nitrogen.

\section{Cell Culture, Stimulation, and Transfection}

Isolation of VICs. Noncalcified human aortic valves $(\mathrm{n}=10)$ were taken from donor hearts during heart transplant or autopsy. Primary aortic VICs were digested as described previously. ${ }^{10}$ In brief, nonleaflet tissues were carefully removed after careful removal of the endothelial layer of the aortic and ventricular aspects, and the leaflets were immersed in $0.25 \%$ trypsin at $37^{\circ} \mathrm{C}$ for $5 \mathrm{~min}$. The tissues were then cut into pieces sized approximately $3 \times 3 \mathrm{~mm}$ for a further 2-hour digestion at $37^{\circ} \mathrm{C}$. The obtained primary VICs were then cultured in growth medium (Dulbecco modified Eagle medium supplemented with penicillin with streptomycin and $10 \% \mathrm{FBS}$ ) at $37^{\circ} \mathrm{C}$ under a $5 \%$ carbon dioxide atmosphere. The purity of the cells was confirmed by microscopic examination and evaluation of the expression of marker proteins (Figure E1).

Stimulation and transfection of VICs. The VICs $\left(1-2 \times 10^{6}\right.$ cells/well) were transfected with 50-nmol/L M-miRNA-30b, 50-nmol/L I-miRNA-30b, and 50-nmol/L miRNA-NC in OPTI-MEMI reduced serum medium (Invitrogen) with Lipofectamine 2000 (Invitrogen) according to the manufacturer instructions. The transfection efficiency was evaluated in a preliminary test. Osteogenesis was further induced for 4 days after transfection by incubation in BMP-2-supplemented osteogenesisinducing medium (minimum essential medium $\alpha$ supplemented with $0.1 \%$ FBS, 50-ng/mL BMP-2, 100-nmol/L dexamethasone, $50-\mu \mathrm{g} / \mathrm{mL}$ ascorbic acid, and 5-mmol/L $\beta$-glycerophosphate). After stimulation, the cells were harvested for further study. Cells treated with miRNA-NC or BMP-2 served as control groups.

\section{miRNA Target Site Prediction}

A search for predicted target mRNAs was performed with the databases TargetScan (http://www.targetscan.org), miRanda (www.miranda-im.org), and PicTar (http://pictar.mdc-berlin.de).

\section{Quantitative Real-Time Polymerase Chain Reaction for Detection of miRNA-30s, Runx2, Smad1, and Caspase-3 Expressions}

For quantitative real-time polymerase chain reaction (qRT-PCR) analysis, total RNA was extracted with TRIzol (Invitrogen). SYBR Green qRTPCR (Takara Bio Inc, Otsu, Japan) was performed with primers published previously (Table E1). For miRNA, qRT-PCR primers were designed and purchased from Gene Pharma (Shanghai, China). Gene expression was normalized to that of U6 or $\beta$-actin for miRNA and mRNA, respectively. The expression of target genes was quantified for 6 replicates by qRT-PCR by using One Step SYBR PrimeScript RT-PCR Kit II (Takara) on an ABI StepOne Real-Time PCR System (Applied Biosystems; Life Technologies Corporation, Grand Island, NY) with conventional protocols. The levels of the target relative to the control were determined by the $2^{-\Delta \Delta \mathrm{CT}}$ method (http://www.appliedbiosystems.com).

\section{Alkaline Phosphatase Activity}

For alkaline phosphatase (ALP) activity assays, the treated cells were washed twice with phosphate-buffered saline solution after incubation, 200- $\mu \mathrm{L}$ lysis buffer was added to the cell layer, and the cells were incubated on ice for 5 minutes. The cell lysate was sonicated for 1 minutes and centrifuged at $1000 \mathrm{~g}$ at $4{ }^{\circ} \mathrm{C}$ for 10 minutes. ALP activity was assayed by a spectrophotometric method. The absorbance of each well at $405 \mathrm{~nm}$ was measured with a microplate reader according to the manufacturer instructions.

\section{Western Blot Analysis}

Individual cell lysates (10 $\mu \mathrm{g} / \mathrm{lane}$ ) were separated by sodium dodecyl sulfate polyacrylamide gel electrophoresis and transferred to nitrocellulose membranes (Millipore Corporation, Billerica, Mass). After incubation in SuperBlock T20 PBS Blocking Buffer (Thermo Fisher Scientific Inc, Waltham, Mass), the membranes were incubated with individual antibodies according to manufacturer instructions. The bound antibodies were detected with a horseradish peroxidase-conjugated secondary antibody diluted 1:5000 (Cell Signaling Technology), visualized with Pierce ECL western blotting substrate (Thermo Fisher Scientific), and digitally imaged with an enhanced chemiluminescence system.

\section{Apoptosis Analysis With Annexin V-Fluorescein Isothiocyanate Staining}

Cells $\left(1 \times 10^{6}\right.$ per well $)$ were cultured in 6 -well plates to $70 \%$ to $80 \%$ confluence. The cells were then treated with osteogenesis-inducing agents, with or without miRNA-30b $(50 \mathrm{nmol} / \mathrm{L})$, for 96 hours. Cells were collected, and the annexin $\mathrm{V}$-fluorescein isothiocyanate staining assay was performed according to the manufacturer instructions (Nanjing Keygen Biotech, Nanjing, China). The collected cells were briefly washed in icecold phosphate-buffered saline solution twice and resuspended in $300 \mu \mathrm{L}$ of $1 \mathrm{X}$ binding buffer containing $5 \mu \mathrm{L}$ annexin $\mathrm{V}$ and $5 \mu \mathrm{L}$ propidium iodide for 30 minutes at room temperature in the dark. After incubation, the cells 
TABLE 1. Demographic and clinical characteristics of the patients $(\mathbf{n}=\mathbf{1 0})$

\begin{tabular}{lc}
\hline \multicolumn{1}{c}{ Parameter } & Value \\
\hline Age (y, mean \pm SD) & $67.7 \pm 5.14$ \\
Sex ratio (male/female) & $5: 5$ \\
Reason for aortic valve replacement (no.) & \\
$\quad$ Valve stenosis & 6 \\
$\quad$ Valve stenosis and insufficiency & 4 \\
Systemic disease (no.) & \\
$\quad$ Diabetes (type 2) & 2 \\
$\quad$ Hypertension & 3 \\
\hline$S D$, Standard deviation.
\end{tabular}

$S D$, Standard deviation.

were analyzed with a FACSCalibur cytometer (BD Becton Dickinson \& Co, San Jose, Calif).

\section{Dual Luciferase Reporter Assay}

Dual luciferase reporter gene construction. The $3^{\prime}$-UTRs of human genes for Runx2 (547 base pairs), Smad1 (557 base pairs), and caspase- 3 (506 base pairs) were cloned with the appropriate primers (listed in Table E2) with a short extension containing cleavage sites for XhoI ( $5^{\prime}$ end) and NotI ( $3^{\prime}$ end). Amplicons were cleaved with XhoI and NotI and cloned between the XhoI and NotI cleavage sites of the pRL-TK vector.

Luciferase assay. 293T cells were selected on the basis of low endogenous expression of miRNA and grown to $85 \%$ to $90 \%$ confluence in white 24-well plates in Dulbecco modified Eagle medium (Invitrogen) supplemented with $10 \%$ FBS, $1 \%$ nonessential amino acids, L-glutamine, and penicillin with streptomycin at $37^{\circ} \mathrm{C}$ under an atmosphere with $5 \%$ carbon dioxide. The cells were transfected with $20 \mathrm{ng}$ of empty pGL3BASIC vector and pRL-TK-3'-UTR reporter for 6 hours in reduced serum and antibiotic-free Opti-MEM with Lipofectamine 2000. The cells were also cotransfected with M-miRNA-30b, I-miRNA-30b, or miRNA-NC. Firefly-luciferase and Renilla-luciferase activities were measured in cell lysates with a Dual-Luciferase Reporter Assay System (Promega Corporation, Madison, Wis) on a Fusion plate reader (PerkinElmer Inc, Waltham, Mass). Firefly-luciferase activity was used for normalization and as an internal control for transfection efficiency.

\section{RESULTS}

\section{miRNA-30b Eliminated During Calcification}

To determine whether miRNA-30 family members are involved in CAVD, we evaluated the expression profile of
miRNA-30s in surgically removed calcified aortic valves (osteogenic markers are shown in Figure E2). Tissue adjacent to the calcification nodules was used as a control (Table E3). the most notably downregulated member of the miRNA-30 family was miRNA-30b $(0.183 \pm 0.613$; Figure 1, A). To evaluate further the role of miRNA-30b in VIC osteogenesis, VICs were treated with osteoblastinducing medium. The miRNA-30b expression significantly decreased to approximately $3 \%$ of the level in the control group $(0.034 \pm 0.006$; Figure $1, B)$. Taken together, these results suggest that miRNA-30b plays a role in the osteoblast differentiation of VICs in vivo as well as in vitro.

\section{Regulation by miRNA-30b of Osteogenesis in VIC Cultures}

To evaluate the impact of miRNA-30b on VIC osteoblast differentiation, VICs were induced to differentiate into osteoblasts after transfection with M-miRNA-30b or I-miRNA-30b. After 4 days of osteoblast induction, the VICs were subjected to an ALP activity assay. BMP-2 triggered an artificial osteoblastic phenotype in VICs that was characterized by ALP activation (Figure 2, A). ALP activity $(0.246 \pm 0.024$ units $/ \mathrm{mg})$ was significantly higher in the I-miRNA-30b group than in the blank control, BMP-2, and miRNA-NC groups $(0.027 \pm 0.001,0.117 \pm 0.007$, and $0.111 \pm 0.012$ units $/ \mathrm{mg}$, respectively); however, this phenotype was obviously inhibited in VICs treated with M-miRNA-30b (0.022 \pm 0.006 units $/ \mathrm{mg})$.

To confirm further the antiosteogenic effect of miRNA$30 \mathrm{~b}$, we evaluated whether miRNA-30b perturbation affected the level of osteocalcin expression, which is a marker for mature osteoblasts. Relative to the other groups, transfection with I-miRNA-30b resulted in increased osteocalcin mRNA expression $(4.203 \pm 0.141$; Figure $2, B)$. In contrast, introduction of M-miRNA-30b significantly repressed osteocalcin mRNA expression (1.049 \pm 0.173$)$. Increased osteocalcin protein levels were confirmed by immunoblot analysis after BMP-2 treatment in VICs transfected with I-miRNA-30b, and this effect was reversed on introduction
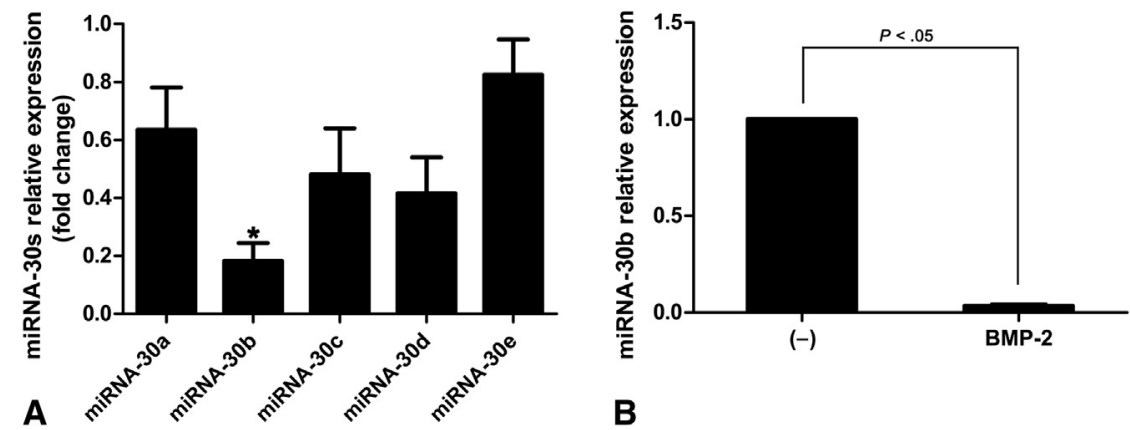

FIGURE 1. MicroRNA-30b (miRNA-30b) is downregulated in osteogenic valve interstitial cells. A, Relative expression levels of microRNA-30 types $($ miRNA-30s) in calcified regions were determined by quantitative real-time polymerase chain reaction $(\mathrm{n}=10$, mean $\pm \mathrm{SD})$. Asterisk indicates $P<.05$. $\mathrm{B}$, MicroRNA-30b levels were significantly reduced in bone morphogenetic protein $2(B M P-2)$-treated cells in 5 independent replications $(P<.05)$. 

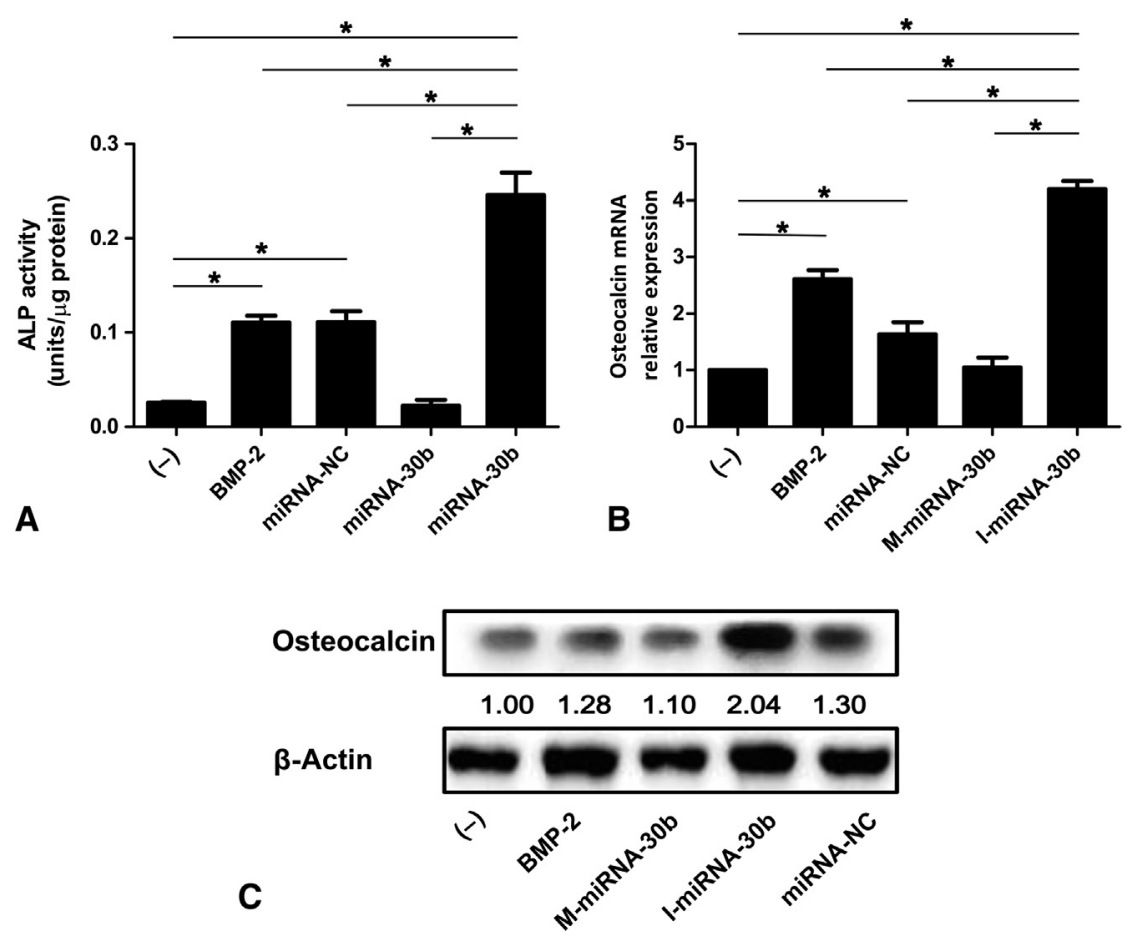

FIGURE 2. MicroRNA-30b inhibits bone morphogenetic protein 2 (BMP-2) signaling-induced osteogenesis in cultured valve interstitial cells. A, Alkaline phosphatase $(A L P)$ activity of 5 independent experiments in each group (mean $\pm \mathrm{SD}$ ). Compared with other groups, microRNA-30b inhibitor (I-miRNA$30 b$ ) activated alkaline phosphatase to a greater extent. Asterisk indicates $P<.05$. B, The levels of osteocalcin messenger RNA ( $m R N A)$ were determined by quantitative real-time polymerase chain reaction (mean $\pm \mathrm{SD}$ ). Inhibition of microRNA-30b upregulated osteocalcin messenger RNA. Asterisk indicates $P<.05$. C, On immunoblotting, miRNA-30b was seen to have an obvious effect on osteocalcin protein expression. miRNA-NC, microRNA negative control; M-miRNA-30b, microRNA-30b mimic.

of M-miRNA-30b (Figure 2, C). These data suggest that M-miRNA-30b and I-miR30b sensitized the VICs to osteogenic signals, thus resulting in a faster or more robust response to osteoinductive soluble factors.

\section{Suppression by miRNA-30b of VIC Apoptosis}

Apoptotic bodies are well known to act as nucleating structures for calcium crystal formation. ${ }^{11}$ We also demonstrated VIC degeneration in CAVD by immunohistochemical staining and terminal deoxynucleotidyl transferase dUTP nick end labeling in sections of calcified valves (Figure E2). To validate the assumption that miRNA-30b in VICs may block calcification by inhibiting apoptosis, VICs were cultured in a low-serum $(0.1 \%)$ medium for 96 hours after transfection. The proportion of apoptotic cells was quantified by annexin V-propidium iodide dual staining. A significant increase in apoptosis was observed in the I-miRNA-30b group (Figure 3). In contrast, the VICs treated with M-miRNA-30b exhibited a reduced proportion of apoptotic cells.

\section{miRNA-30b Attenuates Expressions of Runx2, Smad1, and Caspase-3}

We searched for candidate genes by using the miRNA target prediction databases Target Scan6.2 and PicTar to elucidate further the relationship between miRNA-30b and its targets during calcium nodule formation. The search identified Runx2, Smad1, and caspase-3 as potential target genes for miRNA-30b. Runx2 and Smad1 are widely reported to be key downstream mediators of BMP-2 signaling during bone formation. ${ }^{12,13}$ Caspase- 3 is a major activated cysteine protease reported to function in the early stage of osteoblast proliferation. ${ }^{11,14,15}$

After 96 hours of stimulation in BMP-2-supplemented medium, aortic VICs were evaluated for changes in target gene expression (Figure 4). Relative to the control groups, no effect on mRNA expression of Smad1 was observed. Notably, alteration in miRNA-30b levels led to significant alteration of Runx2 and caspase-3 mRNA levels (Figure 4, $A$ ). Runx2, Smad1, phosphorylated Smad1/5/8, and caspase-3 protein levels were also altered, as demonstrated by Western blot analysis. In particular, the levels of these proteins decreased in cells transfected with M-miRNA-30b and increased in cells subjected to miRNA-30b inhibition by treatment with I-miRNA-30b. (Figure 4, B). Taken together, our results provide concrete evidence that miRNA-30b negatively regulates the expressions of Runx2, Smad1, and caspase 3 in vitro, thereby preventing calcification of VICs.

To examine whether miRNA-30b directly regulates the expressions of Runx2, Smad1, and caspase-3, 293T cells 


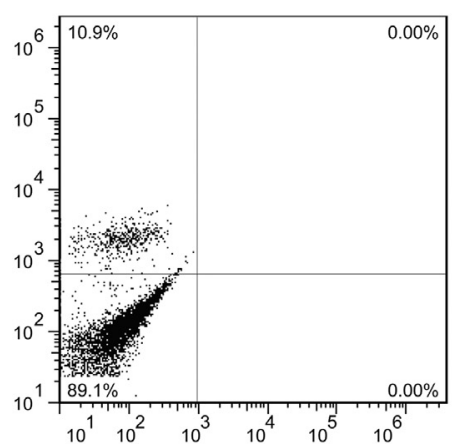

$(-)$

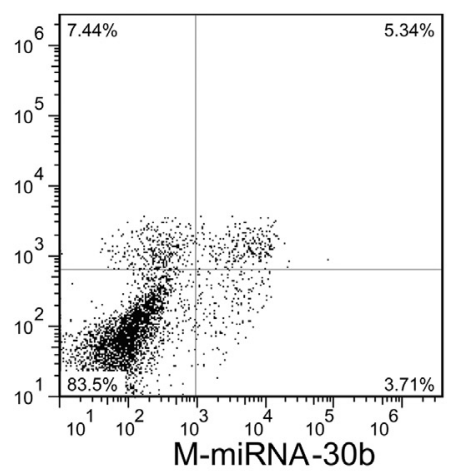

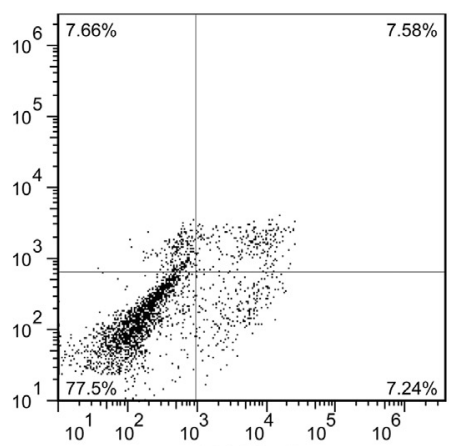

BMP-2

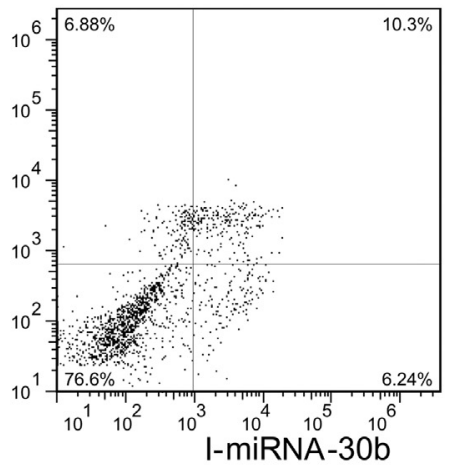

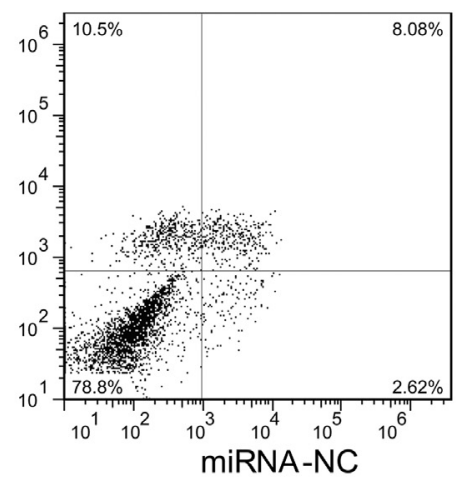

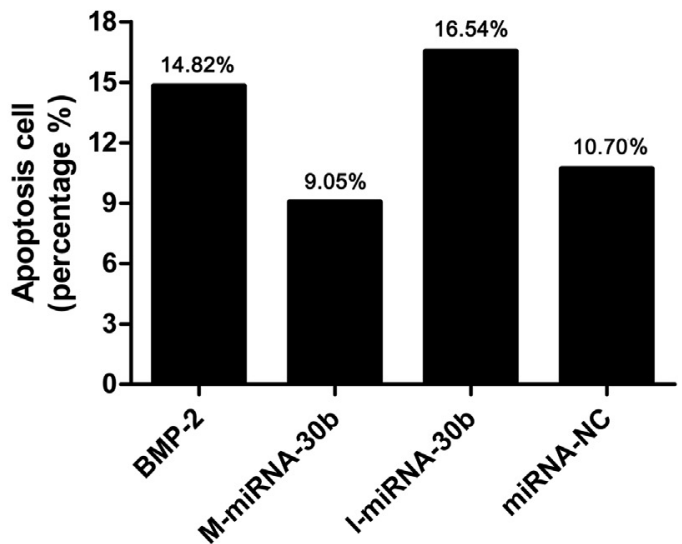

FIGURE 3. MicroRNA-30b suppresses apoptosis in valve interstitial cells. Apoptotic cells are present in the upper right (early stage), and lower right (late stage) quadrants of each panel. The most significant increase in the proportion of apoptotic valve interstitial cells was observed in cells transfected with microRNA-30b inhibitor (I-miRNA-30b). In contrast, valve interstitial cells treated with microRNA-30b mimic (M-miRNA-30b) showed decreased apoptosis. BMP-2, Bone morphogenetic protein 2 ; $m i R N A-N C$, microRNA negative control.

were transfected with a luciferase reporter construct containing the wild-type $3^{\prime}$-UTR of each gene, and miRNA-NC was used as the negative control. The effects of miRNA-30b on reporter expression were determined 24 hours after transfection by measuring the Renillaluciferase signal normalized to firefly-luciferase. Relative to the control cells, Renilla-luciferase activity clearly decreased in cells transfected with M-miRNA-30b and increased in miRNA-30b knockdown cells treated with I-miRNA-30b (Figure 5). As expected, M-miRNA-30b significantly inhibited the activity of the wild-type reporter genes. These data provide evidence that miRNA-30b inhibits the expressions of Runx $2, \mathrm{Smad} 1$, and caspase- 3 by directly binding to distinct sites within the $3^{\prime}$-UTRs of their mRNAs.

\section{DISCUSSION}

In this study, we demonstrated that transfection of M-miRNA-30b into cultured human VICs blocks osteoblastic transformation and apoptosis. Evaluation of the miRNA-30 profile in calcific aortic leaflets revealed a significant downregulation of miRNA-30b in stenotic leaflets, which was associated with increased expressions of Runx2, osteocalcin, and caspase-3. BMP-2-treated VICs also exhibited a dramatic reduction in miRNA-30b level. Furthermore, an on-off experiment suggested that in response to BMP-2, miRNA-30b acts as an effective negative regulator of early osteoblast differentiation through its suppression of Runx2, Smad1, and caspase-3 expressions.

The cellular signals driving aortic valve calcification are reminiscent of those involved in bone formation, and some evidence suggests that the BMP-2 pathway is a central regulator of calcification in CAVD through induction of Runx2 and through the induction apoptosis. ${ }^{16-18}$ Smad1 is phosphorylated and activated by BMP type 1 receptor kinases and thus participates in bone formation and cellular apoptosis. $^{13,19}$ In this study, we observed significantly lower protein expressions of both phosphorylated Smad1/ $5 / 8$ and total Smad1 in the group treated with the M-miRNA-30b. Consequently, both mRNA and protein levels of Runx2 were remarkably lower than those in the miRNA-30b knockdown (I-miRNA-30b) and control groups, which indicates the involvement of BMP-2 signaling. Caspase-3, a major activated cysteine protease of the caspase family, is a pivotal component in the apoptotic process, ${ }^{20}$ and it has been reported to be required for 


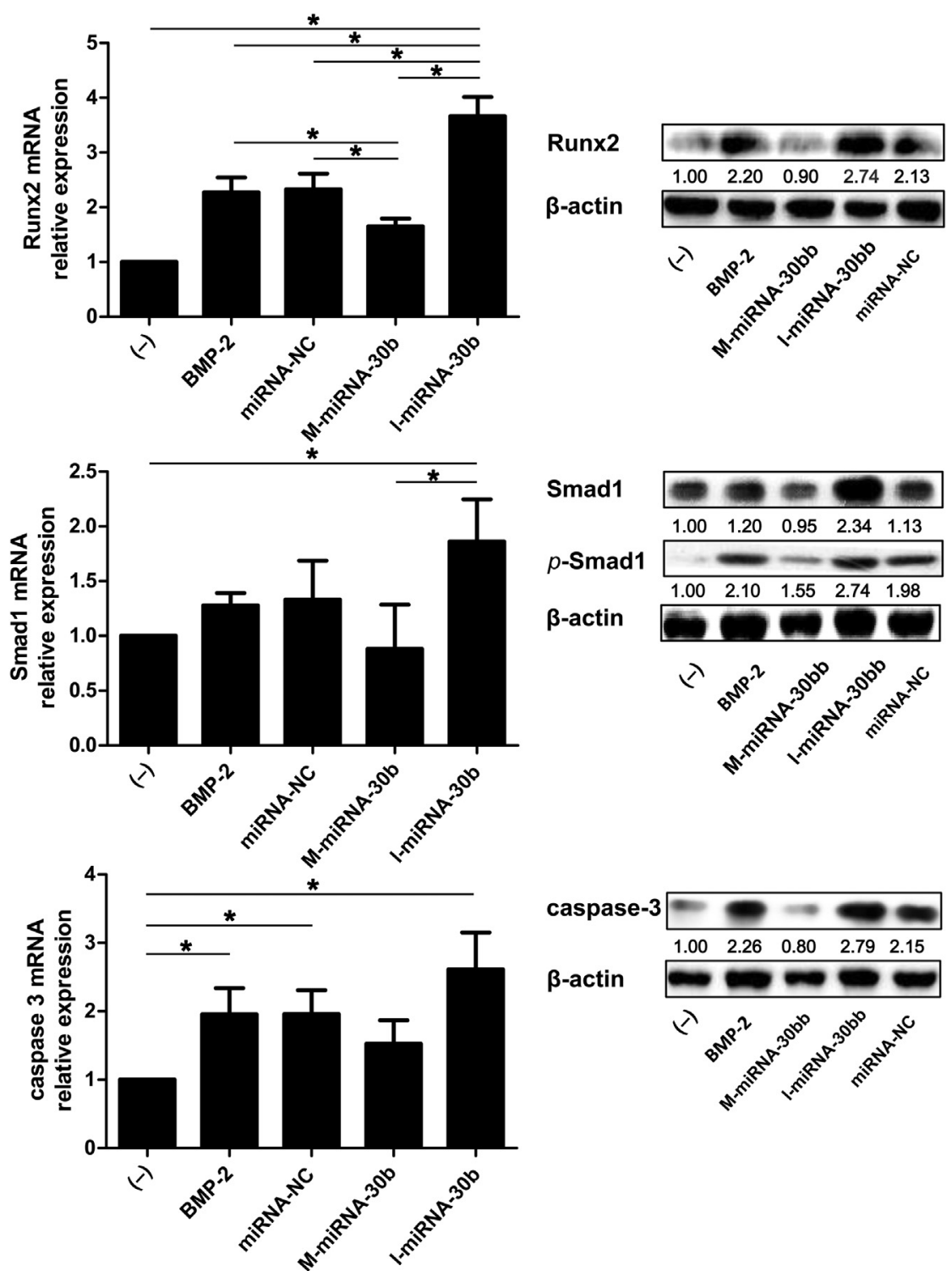

FIGURE 4. MicroRNA-30b attenuates bone morphogenetic protein 2 (BMP-2)-induced osteogenic differentiation in valve interstitial cells. Valve interstitial cells were evaluated with regard to micro RNA-30b. After culture in osteogenic differentiation medium for 4 days, quantitative real-time polymerase chain reaction and Western blotting were performed to evaluate osteogenic differentiation. A, MicroRNA-30b regulated the osteoblast markers Runx2, Smad1, and caspase-3 at the messenger RNA $(m R N A)$ level. Asterisk indicates $P<.05$. B, Western blots for Runx2, Smad1, $p$-Smad1, and caspase-3 were performed with the total cell lysates. miRNA-NC, microRNA negative control; M-miRNA-30b, microRNA-30b mimic; I-miRNA-30b, microRNA$30 \mathrm{~b}$ inhibitor.

maintenance of the proliferative capacity of human bone marrow stromal stem cells during osteogenic differentiation. ${ }^{21}$ In addition, apoptosis has been reported to precede calcification in vascular smooth muscle cells, and apoptotic bodies derived from these cells may act as nucleating structures for calcium crystal formation. ${ }^{11}$ Caspase inhibition has previously been shown in vitro to reduce substantially BMPinduced ALP activation and osteogenesis. ${ }^{22}$ The role of caspase-3 in aortic valve calcification, however, remains poorly understood. Our qRT-PCR and Western blot data demonstrate that transfection of M-miRNA-30b leads to a reduction in caspase-3 level, whereas miRNA-30b knockdown by I-miRNA-30b resulted in increased mRNA and protein levels of caspase-3, relative to the expression levels in the control cells, in addition to decreased VIC apoptosis and ALP activity.

In cancer models, bone formation, autoimmune diseases, and angiogenesis, miRNA-30s are widely involved in the regulation of cell fate decisions. ${ }^{23-27}$ In blood vessels, miRNA-30b and miRNA-30c were found to prevent vascular smooth muscle cell calcification. ${ }^{24}$ In the heart, miRNA$30 \mathrm{~b}$ and 6 other miRNAs showed significant differences in 

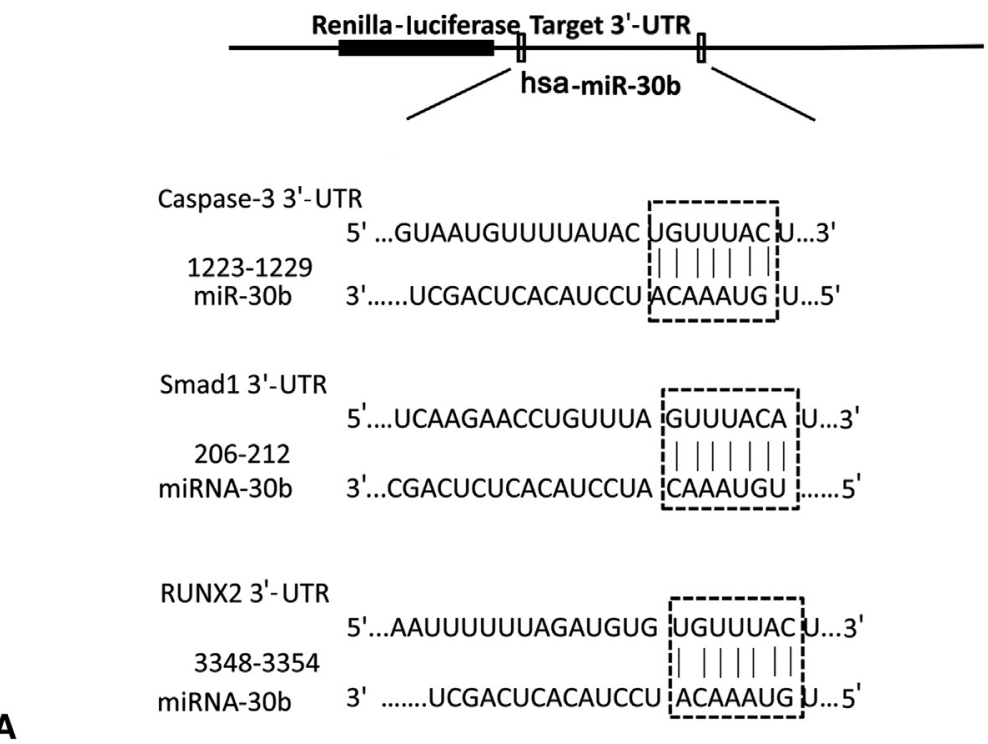

A

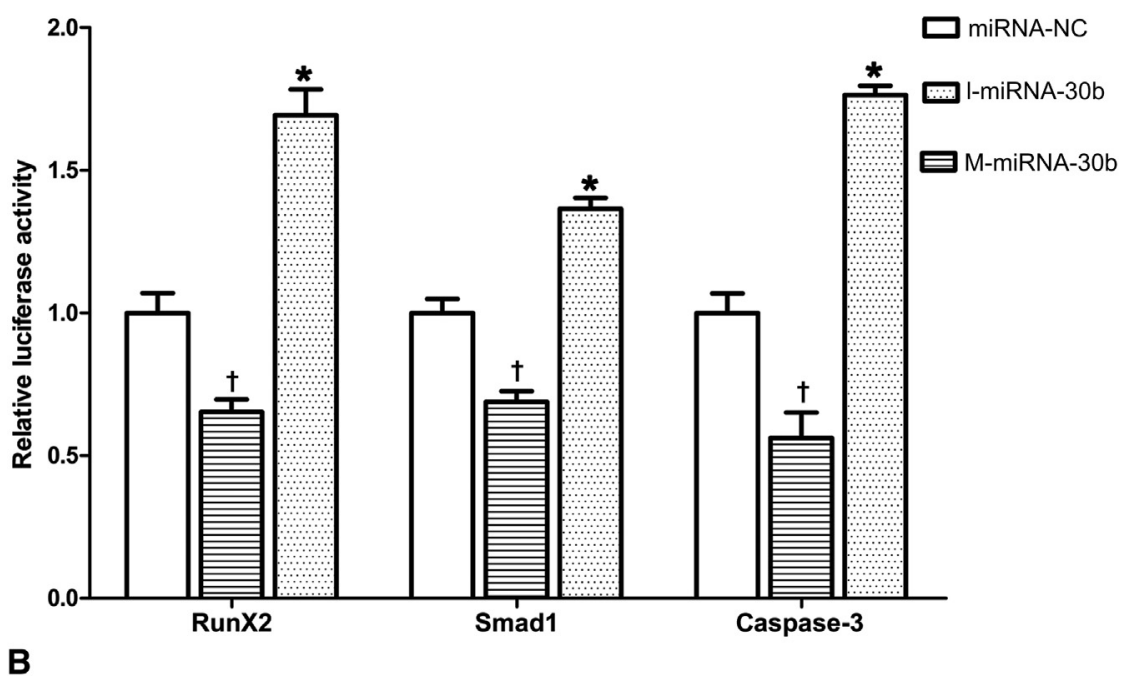

FIGURE 5. Runx2, Smad1, and caspase- 3 are direct targets of microRNA-30b (miRNA-30b). A, Schematic representation of putative target sites for microRNA-30b in the $3^{\prime}$ untranslated regions (UTRs) of human Runx2, Smad1, and caspase-3 messenger RNA. B, Transfection with a micro RNA-30b mimic (M-miRNA-30b) inhibited Renilla-luciferase activity, whereas microRNA-30b knockdown with a microRNA-30b inhibitor (I-miRNA-30b) enhanced it. The Renilla-luciferase signal was normalized against the firefly-luciferase signal. The results are presented as the mean $\pm \mathrm{SD}$ for each group of cells from 4 independent experiments. Dagger and asterisk indicate $P<.05$. miRNA-NC, MicroRNA negative control.

expression between the stenotic and insufficient bicuspid aortic valve phenotypes. ${ }^{28}$ In this study, we first evaluated the miRNA-30s expression profile in patients with degenerative calcification of the aortic valve who did not show congenital valve diseases. We demonstrated that among the miRNA-30s, miRNA-30b exhibited the most dramatic reduction in calcific aortic valves and BMP-2-stimulated VICs. Our gain and loss of function experiments demonstrated decreased ALP activity and osteocalcin expression, which may underlie an antagonizing effect of miRNA-30b against osteoblast formation. Our findings reveal that miRNA-30b attenuates the osteogenic differentiation of
VICs by directly targeting Runx2, Smad1, and caspase-3. We demonstrated for the first time, to the best of our knowledge, that Runx2, Smad1, and caspase- 3 are common targets of miRNA-30b in human beings by examining direct evidence from a dual luciferase reporter assay. In summary, these data suggest that miRNA-30b is a negative regulator that influences both osteocyte formation and cell apoptosis during BMP-2-induced calcification in primary human VICs.

Currently, surgical replacement and transcatheter implantation are the only treatments for aortic stenosis in CAVD. Improved understanding of the underlying pathogenetic 
mechanisms of valvular calcification could lead to the development of targeted treatments to inhibit stenosis development and delay or even eliminate the need for valve replacement. Yanagawa and colleague ${ }^{29}$ have introduced miRNA-141 to cultured porcine VICs to block osteoblastic transformation through a BMP-2-dependent mechanism; however, miR-204 is reported to inhibit osteogenesis by directly targeting Runx $2 .^{6}$ These promising results and our current findings indicate that miRNAs provide a tool for fine-tuning of protein levels. In view of the indispensable role of miRNA-30b in cellular calcification and its multiple target genes, miRNA-30b may have potential therapeutic applications for diseases involving VIC malfunction.

In conclusion, we have demonstrated that in response to BMP-2, miRNA-30b negatively regulates the early calcification of aortic VICs through suppression of Runx2, Smad1, and caspase-3. To the best of our knowledge, this is the first study to demonstrate in vitro a multifunctional regulatory role of miRNA-30b in aortic VICs. Moreover, our findings suggest that miRNA-30b maybe an effective candidate for the prevention and treatment of aortic valve calcification.

\section{Study Limitations}

This study has some limitations. First, the mechanism underlying the change in expression of miRNA-30s during disease processes is still unknown. Second, high-quality RNA was not reliably obtained from severely calcified regions. Third, several other known calcium signaling pathways exist, and miRNAs may affect hundreds of target sequences, so the overall importance of the regulation of BMP-2 by miRNA-30b remains to be explored. Finally, our studies of miRNA-30b-mediated regulation of BMP2-dependent calcification in human VICs have established a potential strategy to prevent aortic valve calcification by targeting Runx2, Smad1, and caspase-3. Additional work is needed to establish a causal relationship between miRNA-30b and calcification.

\section{References}

1. Rajamannan NM, Subramaniam M, Rickard D, Stock SR, Donovan J, Springett M, et al. Human aortic valve calcification is associated with an osteoblast phenotype. Circulation. 2003;107:2181-4.

2. Miller JD, Weiss RM, Heistad DD. Calcific aortic valve stenosis: methods, models, and mechanisms. Circ Res. 2011;108:1392-412.

3. Liu AC, Joag VR, Gotlieb AI. The emerging role of valve interstitial cell phenotypes in regulating heart valve pathobiology. Am J Pathol. 2007;171:1407-18.

4. Moreno PR, Astudillo L, Elmariah S, Purushothaman KR, Purushothaman M, Lento PA, et al. Increased macrophage infiltration and neovascularization in congenital bicuspid aortic valve stenosis. J Thorac Cardiovasc Surg. 2011;142: 895-901.

5. Bartel DP. MicroRNAs: genomics, biogenesis, mechanism, and function. Cell. 2004;116:281-97.
6. Huang J, Zhao L, Xing L, Chen D. MicroRNA-204 regulates Runx2 protein expression and mesenchymal progenitor cell differentiation. Stem Cells. 2010; 28:357-64.

7. Li Z, Hassan MQ, Volinia S, van Wijnen AJ, Stein JL, Croce CM, et al. A microRNA signature for a BMP2-induced osteoblast lineage commitment program. Proc Natl Acad Sci U S A. 2008;105:13906-11.

8. Wu T, Zhou H, Hong Y, Li J, Jiang X, Huang H. miR-30 family members negatively regulate osteoblast differentiation. J Biol Chem. 2012;287:7503-11.

9. Pritchard CC, Cheng HH, Tewari M. MicroRNA profiling: approaches and considerations. Nat Rev Genet. 2012;13:358-69.

10. Filip DA, Radu A, Simionescu M. Interstitial cells of the heart valves possess characteristics similar to smooth muscle cells. Circ Res. 1986;59:310-20.

11. Proudfoot D, SkepperJN Hegyi L, Bennett MR, Shanahan CM, Weissberg PL. Apoptosis regulates human vascular calcification in vitro: evidence for initiation of vascular calcification by apoptotic bodies. Circ Res. 2000;87:1055-62.

12. Chen G, Deng C, Li YP. TGF- $\beta$ and BMP signaling in osteoblast differentiation and bone formation. Int J Biol Sci. 2012;8:272-88.

13. Haÿ E, Lemonnier J, Fromigué O, Marie PJ. Bone morphogenetic protein-2 promotes osteoblast apoptosis through a Smad-independent, protein kinase $\mathrm{C}-\mathrm{de}-$ pendent signaling pathway. J Biol Chem. 2001;276:29028-36.

14. Hoodless PA, Haerry T, Abdollah S, Stapleton M, O'Connor MB, Attisano L, et al. MADR1, a MAD-related protein that functions in BMP2 signaling pathways. Cell. 1996;85:489-500.

15. Fuchs Y, Steller H. Programmed cell death in animal development and disease. Cell. 2011;147:742-58. Erratum in: Cell. 2011;147:1640.

16. Yang X, Meng X, Su X, Mauchley DC, Ao L, Cleveland JC Jr, et al. Bone morphogenic protein 2 induces Runx 2 and osteopontin expression in human aortic valve interstitial cells: role of Smad1 and extracellular signal-regulated kinase 1/2. J Thorac Cardiovasc Surg. 2009;138:1008-15.

17. Balachandran K, Sucosky P, Jo H, Yoganathan AP. Elevated cyclic stretch induces aortic valve calcification in a bone morphogenic protein-dependent manner. Am J Pathol. 2010;177:49-57.

18. Hyzy SL, Olivares-Navarrete R, Schwartz Z, Boyan BD. BMP2 induces osteoblast apoptosis in a maturation state and noggin-dependent manner. J Cell Biochem. 2012;113:3236-45.

19. Wang M, Jin H, Tang D, Huang S, Zuscik MJ, Chen D. Smad1 plays an essential role in bone development and postnatal bone formation. Osteoarthritis Cartilage. 2011;19:751-62.

20. Nicholson DW. Caspase structure, proteolytic substrates, and function during apoptotic cell death. Cell Death Differ. 1999;6:1028-42.

21. Miura M, Chen XD, Allen MR, Bi Y, Gronthos S, Seo BM, et al. A crucial role of caspase- 3 in osteogenic differentiation of bone marrow stromal stem cells. J Clin Invest. 2004;114:1704-13.

22. Mogi M, Togari A. Activation of caspases is required for osteoblastic differentiation. J Biol Chem. 2003;278:47477-82.

23. Shao C, Yu Y, Yu L, Pei Y, Feng Q, Chu F, et al. Amplification and up-regulation of microRNA-30b in oral squamous cell cancers. Arch Oral Biol. 2012;57: 1012-7.

24. Balderman JA, Lee HY, Mahoney CE, Handy DE, White K, Annis S, et al. Bone morphogenetic protein-2 decreases microRNA-30b and microRNA-30c to promote vascular smooth muscle cell calcification. J Am Heart Assoc. 2012;1: e003905.

25. Bridge G, Monteiro R, Henderson S, Emuss V, Lagos D, Georgopoulou D, et al. The microRNA-30 family targets DLL4 to modulate endothelial cell behavior during angiogenesis. Blood. 2012;120:5063-72.

26. Hafez MM, Hassan ZK, Zekri AR, Gaber AA, Al Rejaie SS, Sayed-Ahmed MM, et al. MicroRNAs and metastasis-related gene expression in Egyptian breast cancer patients. Asian Pac J Cancer Prev. 2012;13:591-8.

27. Liu Y, Dong J, Mu R, Gao Y, Tan X, Li Y, et al. MicroRNA-30a promotes B cell hyperactivity in patients with systemic lupus erythematosus by direct interaction with Lyn. Arthritis Rheum. 2013;65:1603-11.

28. Nigam V, Sievers HH, Jensen BC, Sier HA, Simpson PC, Srivastava D, et al. Altered microRNAs in bicuspid aortic valve: a comparison between stenotic and insufficient valves. J Heart Valve Dis. 2010;19:459-65.

29. Yanagawa B, Lovren F, Pan Y, Garg V, Quan A, Tang G, et al. miRNA-141 is a novel regulator of BMP-2-mediated calcification in aortic stenosis. $J$ Thorac Cardiovasc Surg. 2012;144:256-62. 

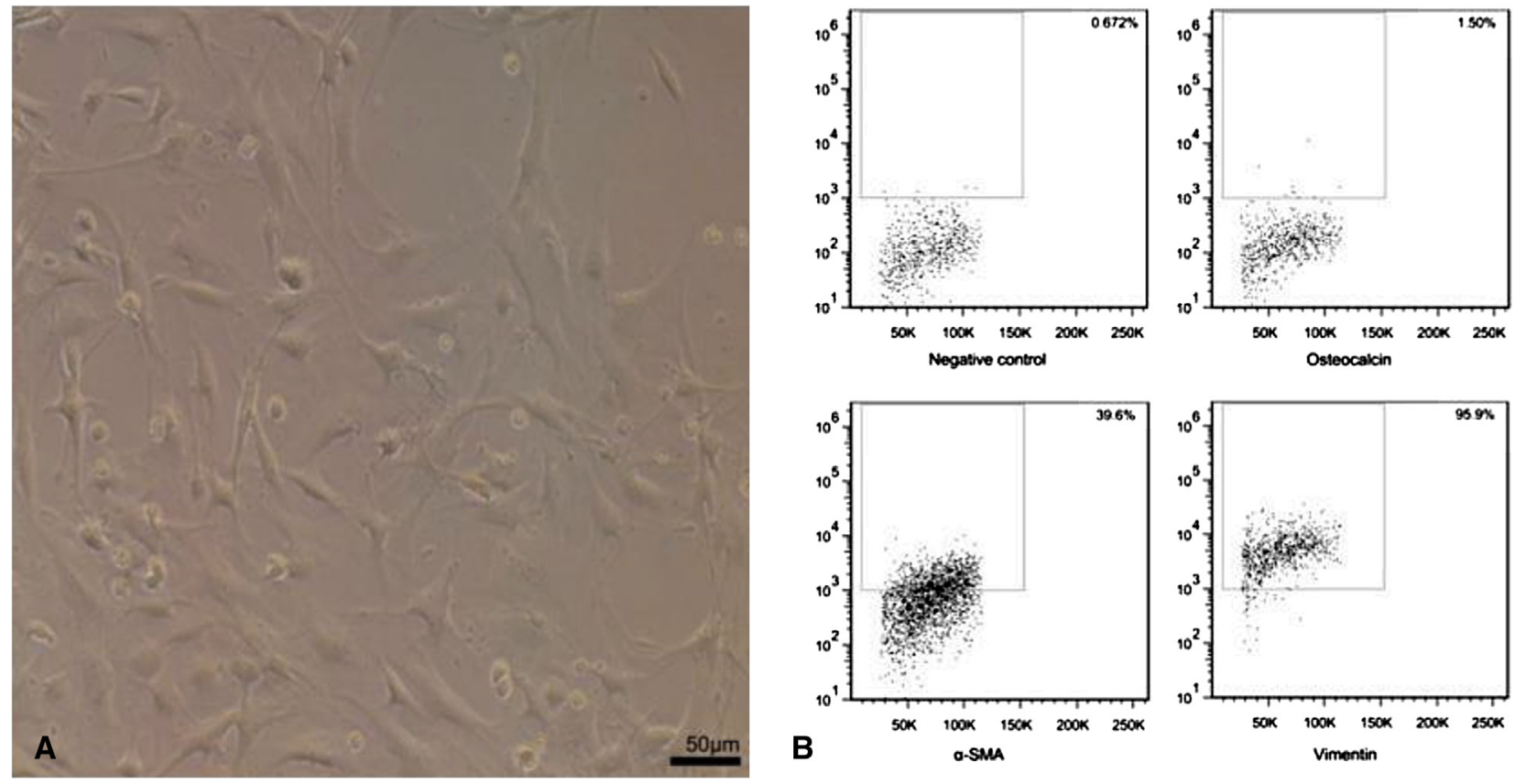

FIGURE E1. Identification of the primary aortic valve interstitial cells. A, Microscopic analysis of cell morphology. B, The primary aortic valve interstitial cells express specific markers ( $\alpha$-SMA $40 \%$, vimentin $96 \%$ ).
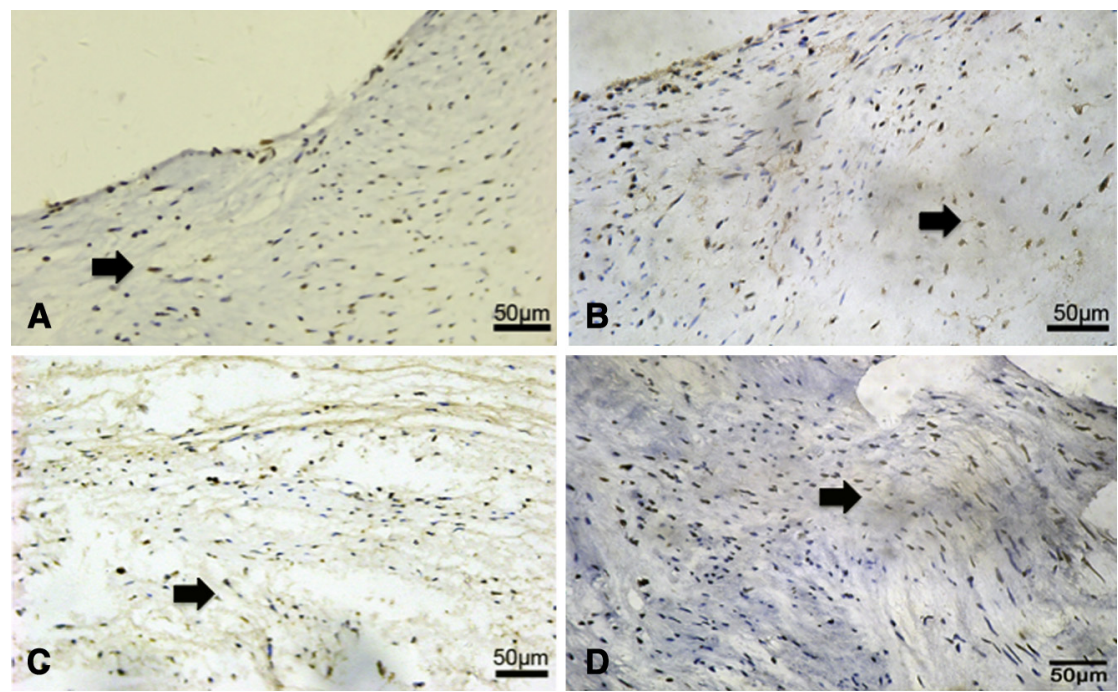

FIGURE E2. Histopathologic staining of the calcified aortic valves. Arrows indicate the positive immunohistochemical staining regions for Runx2 (A), osteocalcin (B), caspase-3 (C), and terminal deoxynucleotidyl transferase dUTP nick-end labeling (D) in the high-magnification view (original magnification $\times 200)$. Bar represents $50 \mu \mathrm{m}$. 
TABLE E1. Primers for quantitative real-time polymerase chain reaction

\begin{tabular}{lll}
\hline \multicolumn{1}{c}{ Gene } & \multicolumn{1}{c}{ Forward } & \multicolumn{1}{c}{ Reverse } \\
\hline$\beta$-Actin & $5^{\prime}$-AACAG CCGCCTAGAAGCAC-3' & $5^{\prime}$-CGTTGACATCCGTAAAGACC-3' $^{\prime}$ \\
Runx2 & $5^{\prime}$-AGCTTCTGTCTGTGCCTTCTGG-3' & $5^{\prime}$-GGA GTAGA GAGGCAAGAGTT T-3' $^{\prime}$ \\
Smad1 & $5^{\prime}$-TTCCATGCCTCCTCCACAAG-3' & $5^{\prime}$-AGGCATTCGGCATACACCTC-3' \\
Caspase-3 & $5^{\prime}$-AGCGAATCAATGGACTCTGG-3' & $5^{\prime}$-CCGAGATGTCATTCCAGTGC- $3^{\prime}$ \\
Osteocalcin & $5^{\prime}$-CTT TGT GTC CAA GCA GGA-3' & $5^{\prime}$-CTGAAAGCCGATGTGGTCAG-3' \\
U6 & $5^{\prime}$-GTGCTCGCTTCGGCAGCACATATAC-3' & $5^{\prime}$-AAAAATATGGAACGCTTCACGAATTTG-3' \\
\hline
\end{tabular}

TABLE E2. Primers for target gene $3^{\prime}$-UTRs

\begin{tabular}{lll}
\hline \multicolumn{1}{c}{ Gene } & \multicolumn{1}{c}{ Forward } & \multicolumn{1}{c}{ Reverse } \\
\hline Runx2 & 5'CTAGTCTAGACCACACCATTAGGGACCATC-3' $^{\prime}$ & 5'-ATAAGAATGCGGCCGCATCATGACCTGTGGCACAAA-3' $^{\prime}$ \\
Smad1 & 5'CTAGTCTAGAGCATCTGCCTCTGGAAAACT-3' $^{\prime}$ & 5'-ATAAGAATGCGGCCGCTGACAGCAAGTATGGTCAGCA-3' $^{\prime}$ \\
Caspase-3 & 5'-CTAGTCTAGATAAATGAATGGGCTGAGCTG-3' $^{\prime}$ & 5'ATAAGAATGCGGCCGCCAACAAGAAATCTCCCTGAA-3' $^{\prime}$ \\
\hline
\end{tabular}

TABLE E3. Measurement of microRNA-30 family in calcified valve

\begin{tabular}{cccc}
$\begin{array}{c}\text { miRNA-30 } \\
\text { family }\end{array}$ & $\begin{array}{c}\boldsymbol{\Delta} \mathbf{C T}_{\text {calcific }} \\
\text { regions }\end{array}$ & $\begin{array}{c}\boldsymbol{\Delta} \mathbf{C T} \text { control } \\
\text { segments }\end{array}$ & $\begin{array}{c}\boldsymbol{\Delta} \mathbf{\Delta C T}\left(\boldsymbol{\Delta} \mathbf{C T}_{\text {calcific regions }}-\right. \\
\left.\mathbf{\Delta} \mathbf{C T}_{\text {control segments }}\right)\end{array}$ \\
\hline miRNA-30a & $5.37 \pm 1.92$ & $4.68 \pm 1.91$ & $0.69 \pm 0.37$ \\
miRNA-30b & $5.86 \pm 0.47$ & $3.31 \pm 0.63$ & $2.55 \pm 0.62$ \\
miRNA-30c & $6.11 \pm 1.30$ & $4.99 \pm 0.92$ & $1.12 \pm 0.47$ \\
miRNA-30d & $6.63 \pm 0.85$ & $5.30 \pm 0.71$ & $1.33 \pm 0.49$ \\
miRNA-30e & $5.72 \pm 0.79$ & $5.43 \pm 0.69$ & $0.30 \pm 0.20$ \\
\hline
\end{tabular}

$\Delta \mathrm{CT}=\mathrm{CT}$, target $-\mathrm{CT}$, endogenous control and $\Delta(\Delta \mathrm{CT})=\Delta \mathrm{CT}$, stimulated -

$\triangle \mathrm{CT}$, control. miRNA, MicroRNA. 